\title{
Double-Orifice Mitral Valve: An Educational Presentation
}

Minna Moreira Dias Romano', MD, PhD; Antonio Carlos Menardi², MD, PhD; Osvaldo César Almeida-Filho' , MD, PhD; Walter Vilella de Andrade Vicente², MD, PhD; Paulo Roberto Barbosa Evora², MD, PhD

\begin{tabular}{|c|c|}
\hline \multicolumn{2}{|c|}{ eviatio } \\
\hline DOVM & $=$ Double-orifice mitral valve \\
\hline ASD & $=$ Atrial septal defect \\
\hline TEE & = Transesophageal echocardiogram \\
\hline TTE & $=$ Transthoracic echocardiogram \\
\hline
\end{tabular}

A 59-year-old male patient with history of aortic coarctation correction surgery at age 11 presented at the emergency room with severe inspiratory dyspnea and mitral systolic murmur. He was a patient without follow-up after surgical correction of aortic coarctation until the appearance of increasing dyspnea in the last 2 years. The first possible diagnosis of double-orifice mitral valve (DOVM) was based on echocardiogram data performed in 2012 as part of the investigation of increasing dyspnea. The recent transesophageal echocardiogram (TEE) revealed normal ventricular function, moderate to severe mitral regurgitation, DOMV and an 11-mm ostium secundum atrial septal defect (ASD).
${ }^{1}$ Cardiology Center of the Department of Internal Medicine, Faculdade de Medicina de Ribeirão Preto da Universidade de São Paulo (FMRP-USP), Ribeirão Preto, SP, Brazil.

${ }^{2}$ Department of Surgery and Anatomy, Faculdade de Medicina de Ribeirão Preto da Universidade de São Paulo (FMRP-USP),

Ribeirão Preto, SP, Brazil.

This study was carried out at the Department of Surgery and Anatomy, Faculdade de Medicina de Ribeirão Preto da Universidade de São Paulo (FMRP-USP), Ribeirão Preto, SP, Brazil.
Correspondence Address:

Paulo Roberto Barbosa Evora

iD https://orcid.org/0000-0001-9631-946X

Faculdade de Medicina de Ribeirão Preto da Universidade de São Paulo Department of Surgery and Anatomy

Av. Bandeirantes, 3900 - Ribeirão Preto, SP, Brazil - Zip code: 14048-900

E-mail: prbevora@gmail.com 

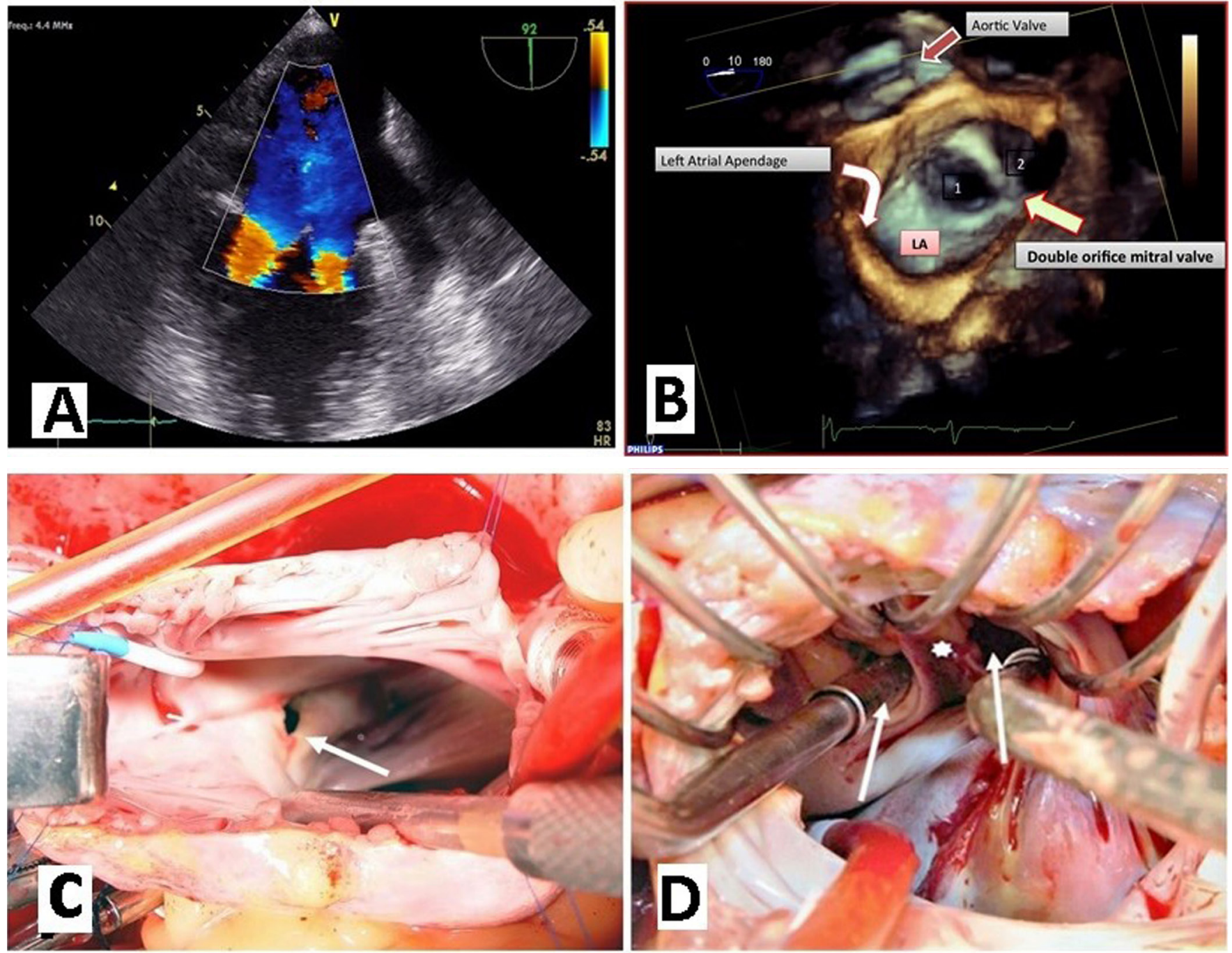

Fig. 1 - A) 2D echocardiogram showing DOMV; B) 3D echocardiogram showing DOMV; C) Intraoperative ASD; D) DOMV shown by an atrial septostomy (the arrows denote two mitral valve orifices and the septum between the two mitral orifices).

\section{Questions}

A. What is the cause of mitral valve insufficiency?

B. What would be considered as a clinical challenge?

C. Regarding the current imaging techniques, which would be the most adequate for the diagnosis of DOMV?

D. What about the surgical treatment challenges?

\section{Discussion of Questions}

Double-orifice mitral valve is usually associated with other cardiac anomalies, so, it is supposed that DOMV is a congenital valve disease (Question A).

There are no specific clinical signs suggestive of DOMV. DOMV is usually an incidental finding in the diagnosis of the elderly patient and may be missed or undiagnosed in asymptomatic or even symptomatic patients. Most patients stay in normal sinus rhythm, but patients with significantly dysfunctional DOMV may present with heart failure, requiring initial medical therapy. The isolated cases of DOMV do not need therapy and can be followed up only by echocardiographic examinations. However, clinic manifestation and management depend not only on the severity of mitral valve dysfunction, but also on associated malformations, which cause pulmonary hypertension due to pulmonary hyperflow from intracardiac shunt (Question B).

Transthoracic echocardiogram, especially in parasternal short-axis views, is a reliable method and in most cases, sufficient to confirm a diagnosis of DOMV and to determine its type. 3D transthoracic echocardiogram is a diagnostic imaging method complementary to 2D transthoracic echocardiogram. Combining 2D and 3D transthoracic echocardiogram is extremely beneficial in the complete cardiac evaluation and management of DOMV (Question C). 
In a severely stenotic DOMV, percutaneous balloon mitral valvuloplasty has been described, but the experience is limited. In most cases, the dividing bridge is composed of mitral and chordal tissue. Surgical transection of the dividing bridge is not advised in order to avoid iatrogenic mitral regurgitation. Therefore, a valve prosthesis is the most reliable surgical option (Question D).

\section{Brief Consideration of the Case Reported}

As the evaluation of dyspnea was mandatory for a possible surgery indication, the diagnosis was supplemented by ergospirometry and cardiac catheterization to better define the cause of dyspnea, clarified that the patient did not present pulmonary arteriolar hypertension and that pulmonary artery pressure was elevated by the presence of mitral valve insufficiency an d AS D. Th en the su rgery wa s in dicated. An atrial septostomy allows easy surgical access to the mitral valve apparatus that presented a duplicate mitral valve, with two separate mitral valve annuli, each with its own set of leaflets and subvalvular apparatus. It was implanted a $33 \mathrm{M}$ bovine pericardium prosthesis. Unfortunately, it was not possible to preserve the papillaries due to their irregular implantation. The immediate postoperative period was characterized by the difficulty of discontinuing respiratory care. The patient died after about 45 days, due to hospital pneumonia that evolved to septic shock ${ }^{[1-7]}$.

\section{Authors' roles \& responsibilities}

MMDR Substantial contributions to the conception or design of the work; final approval of the version to be published

ACM Substantial contributions to the conception or design of the work; final approval of the version to be published

OCAF Substantial contributions to the conception or design of the work; final approval of the version to be published

WVAV Substantial contributions to the conception or design of the work; final approval of the version to be published

PRBE Substantial contributions to the conception or design of the work; final approval of the version to be published

\section{Learning Points}

- DOMV is usually discovered in childhood or early adolescence because of its association with other congenital heart defects.

- The incidence of the diagnosis of DOMV in the adult tertiary referral echocardiographic laboratory is $0.06 \%$.

- DOMV is usually an incidental finding in the diagnosis of the elderly patient, and's diagnosis may be missed or undiagnosed in asymptomatic or even symptomatic patients.

- DOMV as a cause of symptomatic mitral valve disease is also seen in middle-aged/elderly people. Most patients remain in normal sinus rhythm.

- TTE examination, especially in short-axis parasternal views, is a reliable and, in most cases, sufficient method to confirm the diagnosis of DOMV and to determine its type. However, threedimensional TTE should be used to better delineate and study the anomaly.

\section{ACKNOWLEDGEMENTS}

FAEPA, CNPq, FAPESP.

\section{No financial support. \\ No conflict of interest.}

\section{SELECTED REFERENCES}

1. Anwar AM, McGhie JS, Meijboom FJ, Ten Cate FJ. Double orifice mitral valve by real-time three-dimensional echocardiography. Eur J Echocardiogr. 2008;9(5):731-2. doi: 10.1093/ejechocard/jen149.

2. Liu S, Ren W, Ma C, Yang J. Congenital Double-Orifice Mitral Valve in Asymptomatic Patients. Int Heart J. 2018 Jan 27;59(1):213-215. doi: 10.1536/ ihj.17-033.

3. PillaiW, Karunakaran J. Repair of Double Orifice Left AVValve (DOLAW) with Endocardial Cushion Defect in Adult. Braz J Cardiovasc Surg. 2017;32(4):33840. doi: 10.21470/1678-9741-2016-0034.

4. Pires N, Li W, Senior R, Khattar RS. Incidental finding of a double orifice mitral valve in an elderly patient: value of $3 \mathrm{D}$ imaging. Echo Res Pract. 2017;4(3):K21-K24. doi: 10.1530/ERP-17-0023.

5. Samiei N, Dehghan H, Pourmojib M, Mohebbi A, Hosseini S, Rezaei Y. Isolated double-orifice mitral valve in a young girl. ARYA Atheroscler. 2017;13(6):295-8.

6. Sinha SK, Mishra V, Singh K, Asif M, Sachan M, Kumar A, et al. Bi-Luminal Mitral Valve: Incidence, Clinical Features, Associated Anomaly and Echocardiographic Evaluation. J Clin Med Res. 2016 Dec;8(12):893-898.

7. Tomita Y, Yasui H, Tominaga R. Mitral valve repair for isolated doubleorificemitral valve with torn chordae. Ann Thorac Surg. 1997;64(6):1831-4. 